\title{
A Forest Fire Monitoring and Early Warning System Based on the Technology of Multi-sensor and Multilevel Data Fusion
}

\author{
Zhifu Gao ${ }^{a}$, *Linsheng Huang ${ }^{b}$ \\ School of Electronics and Information Engineering, Anhui University, Hefei, 230601, China \\ aemail: Ilyahu@163.com, bemail:linsheng0808@163.com* (Corresponding author)
}

Keywords: forest fire; multi-sensor; multilevel data fusion; CC2530 SOC; monitoring center

\begin{abstract}
The forest is deemed as a precious and indispensable ecological resource, but forest fire which is of common occurrence all over the world can destroy forest resource and threaten human-living environment. It is urgent for Forest Fire Prevention Departments to adopt a monitoring system that can predict the occurrence and spread of forest fire so as to reduce the loss caused by forest fire. This paper illustrates a monitoring system for forest fire based on multi-sensor and 4G network. The hardware design and software implementations of the monitoring system are presented based on ZigBee technology and 4G communication technology. CC2530 SOC is chosen to acquire the data and send it to $4 \mathrm{G}$ network through which the data is transmitted to the remote monitoring server. By means of multi-sensor and multilevel data fusion, the system accomplishes forest fire early warning according to forest fire rating.
\end{abstract}

\section{Introduction}

The forest is deemed as one of the most precious and indispensable resource, which protects the ecological balance of the earth[1]. However, forest fire hazards ecosystems and causes the loss of human life and property[2]. While the traditional forest fire monitoring and early warning methods are manly based on ground patrolling, watching tower, aerial patrolling, long-distance video monitoring, satellite monitoring[3], they are often prone to miss the best time to extinguish the fire. We can greatly reduce, or even avoid the occurrence of forest fires if we make effective monitoring and early warning.

The occurrence of forest fire is not only related to climate changes, fuel types and human activities, but also closely to the evolution of celestial bodies[4]. Since forest fire occurs with randomness, zoning and forecast fire danger rating is the estimate for potential forest fire occurrence, as a result to predict the occurrence of forest fires every time is impossible. However, it must have its specific external environment and internal conditions in the event of forest fires. Therefore, the scientific analysis of the conditions and rules of forest fire occurrence, timely prediction of the degree of forest fire danger rating, especially the possibility of fire forest fire occurred in people and early warning in the development tendency of fire and forecast issued in time to take the relevant specific measures to reduce the loss of forest fires and the resulting is of great significance for the protection of forest resources.

With the advance of wireless communication technology, wireless sensor network has been paid great attention in all fields [5]. In order to effectively prevent forest fire, this paper designs a forest fire monitoring system based on multilevel data fusion of multi-sensor and $4 \mathrm{G}$ network through which monitoring system can make it easy to implement the connection of monitoring field with remote monitoring server.

\section{Architecture of Monitoring System}

A forest fire monitoring and early warning system based on the technology of multi-sensor and multilevel data fusion is mainly made up of four parts: distributed wireless sensor network, remote wireless data transmission platform, monitoring server as shown as Fig.1. 


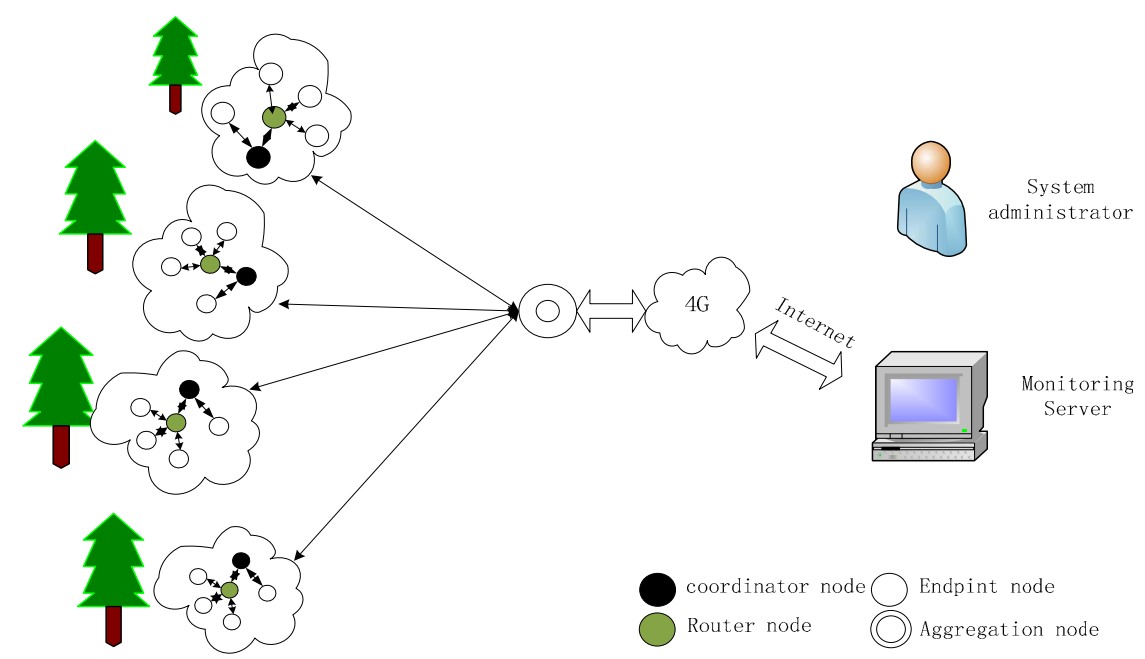

Fig.1.System architecture

\section{A. Distributed wireless sensor network}

Distributed wireless sensor network consists of endpoint nodes, router nodes and coordinating nodes. A large number of endpoint nodes are deployed within or near to the monitoring region to form multilevel hierarchical clustering protocol monitoring network by self-organized and to collect real-time environmental factors such as temperature, humidity, light intensity, smoke concentration and flame. Cluster nodes transmit environmental factors to the cluster head, which deduces a fire rating through fusing the multi-sensor's data and send the fire rating to coordinator by multi-hop routing. The coordinator node is responsible for the data collection, deeper hierarchical data fusing and transfers them to the $4 \mathrm{G}$ network[6].

B. Remote wireless data transmission platform

This paper designs a remote data transmission platform using the communications technology of $4 \mathrm{G}$ that can communicate steadily, which can be in favor of fire-fighting overall scheduling and measures from the forest fire command center.

\section{Monitoring server}

Monitoring server mainly consists of network server with a public IP address, a database and the graphical interface. The monitoring system will trigger an alarm automatically when potential fire hazards or forest fire are found, and help the administrative department of the forest to adopt the effective measures to stop fire hazards stretching in the forest.

\section{Design of System Hardware}

\section{A. Endpoint/router node}

This paper chooses CC2530 SOC which is in favor of Z-Stack produced by TI as the core chip of endpoint node. The endpoint mainly consists of data collection module, data processing module data transmission module and energy management module as shown as Fig.2. Data collection module contains temperature sensor, humidity sensor, light sensor, smoke sensor and flame sensor. The hardware architecture of the router node is the same with endpoint node. 


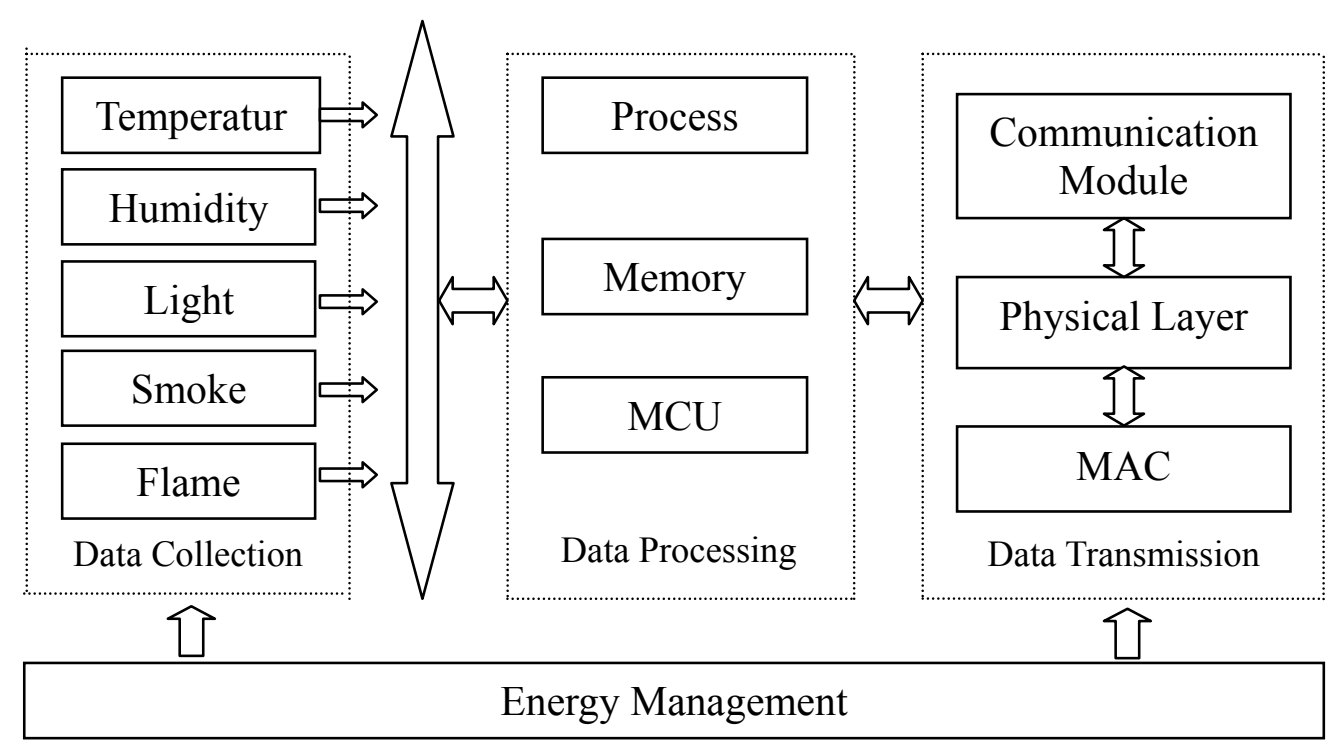

\section{B. Coordinator node}

Fig.2. Structure of endpoint/router node

The coordinator corresponds with $4 \mathrm{G}$ module besides communicating with other network nodes, which is responsible for deeper hierarchical data fusing[7]. This paper chooses CC2530 SOC which is in favor of Z-Stack as the core chip of coordinator node. The coordinator node mainly consists of data collection module, data processing module data transmission module and energy management module as shown as Fig.3. Data collection module contains temperature sensor, humidity sensor, light sensor, smoke sensor and flame sensor.

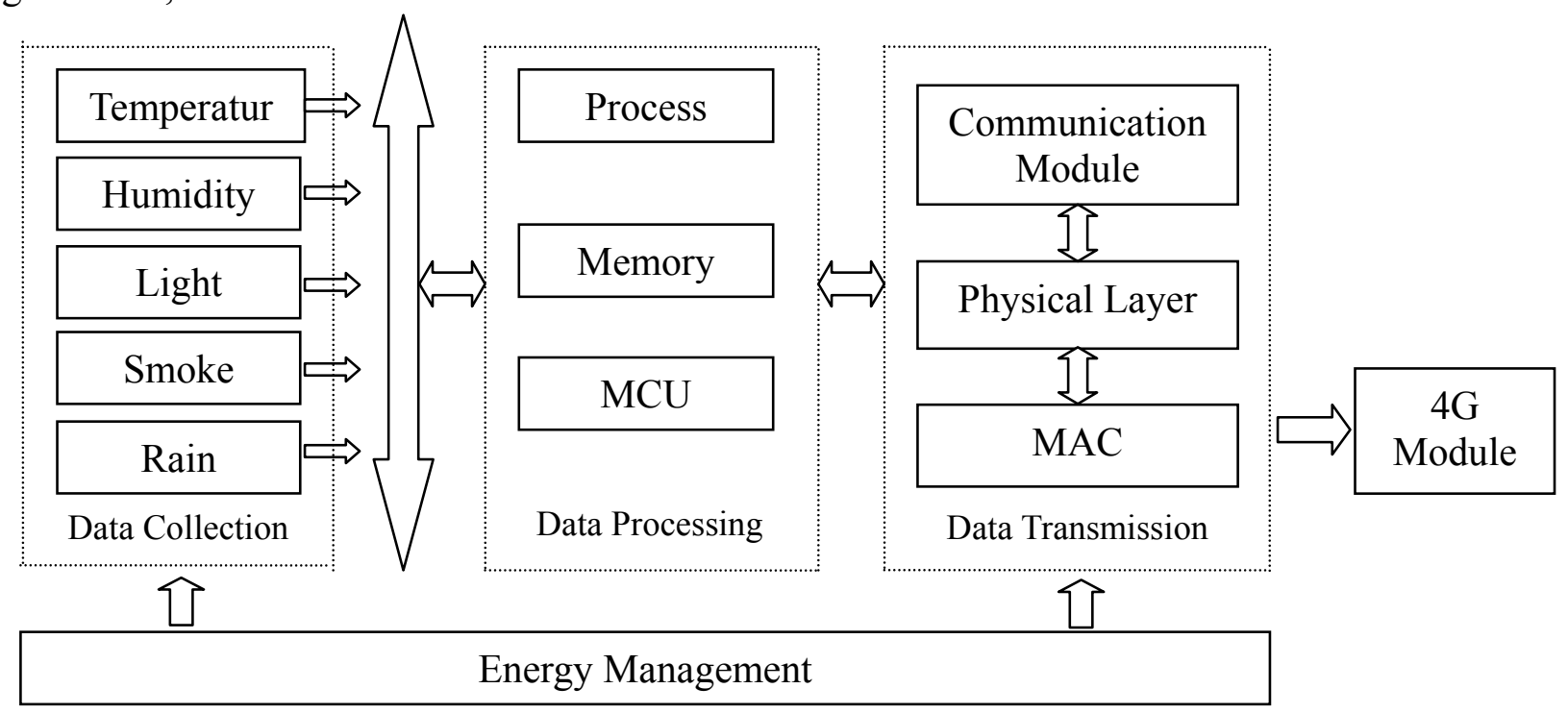

\section{C. $4 G$ Gateway}

Fig.3. Structure of coordinator node

$4 \mathrm{G}$ is the fourth generation of mobile communication technology which could collect WLAN and 3G. Download speed of 4G system could reach to $100 \mathrm{Mbps}$ which is almost 2000 times as fast as Dial-up Internet. At the same time, it is able to meet almost all the users requirements for wireless services[8]. In addition, the $4 \mathrm{G}$ and the DSL could be set in the place where cable modem are not be covered, and then extend to the whole region.

\section{Design of System Software}

The design of system software contains implementation of multilevel hierarchical clustering protocol and data transceiver via $4 \mathrm{G}$ module as well as server interface.This paper mainly talks about the implementation of multilevel hierarchical clustering protocol. 


\section{A. Implementation of multilevel hierarchical clustering protocol}

Multilevel hierarchical clustering protocol which is based on LEACH is a distributed algorithm. This scheme is built for homogenous networks, where all nodes start with the same initial energy. The network operation in MLHC is divided into rounds, each round consists of two stages with two phases for each stage. Each stage is corresponding to a clustering level. The first level of clustering occurs in the first stage, and the network is divided into clusters with a cluster head for each cluster in the same way as in [9]. The cluster heads perform another clustering or a second level of clustering between themselves in the second stage.

\section{B. Design of endpoint node software}

The task of endpoint nodes mainly contains real-time acquiring fire factors of forest environment, gathering information and sending them to the cluster heads. The endpoint nodes will be initialized when the power on, and the timer will be set interrupt. Endpoint nodes enter into low power mode and wait for being awakened, while the processor is in the sleep state, but SPI port and interrupt system will still continue to work, and is always ready to accept system interrupt request[10].

Endpoint nodes will continue to work, acquire fire factors and send them to the cluster heads when the system transmits a signal of acquisition request or the timer's interrupt arrives. Endpoint nodes will enter into sleep mode again until next timer interrupt or external interrupt arrives, which can reduce energy loss.

\section{Design of coordinator node software}

The formation of the network is completed by coordinator node after its initialization. The coordinator node will transmit data after the network is built as shown as Fig.4. When the data that monitored by multi-sensors arrives, the coordinator will analysis data packet, and re-packs data in corresponding with the required format, then sends packaged data to the $4 \mathrm{G}$ module via the serial port, $4 \mathrm{G}$ module is responsible for sending the data to the monitoring server.

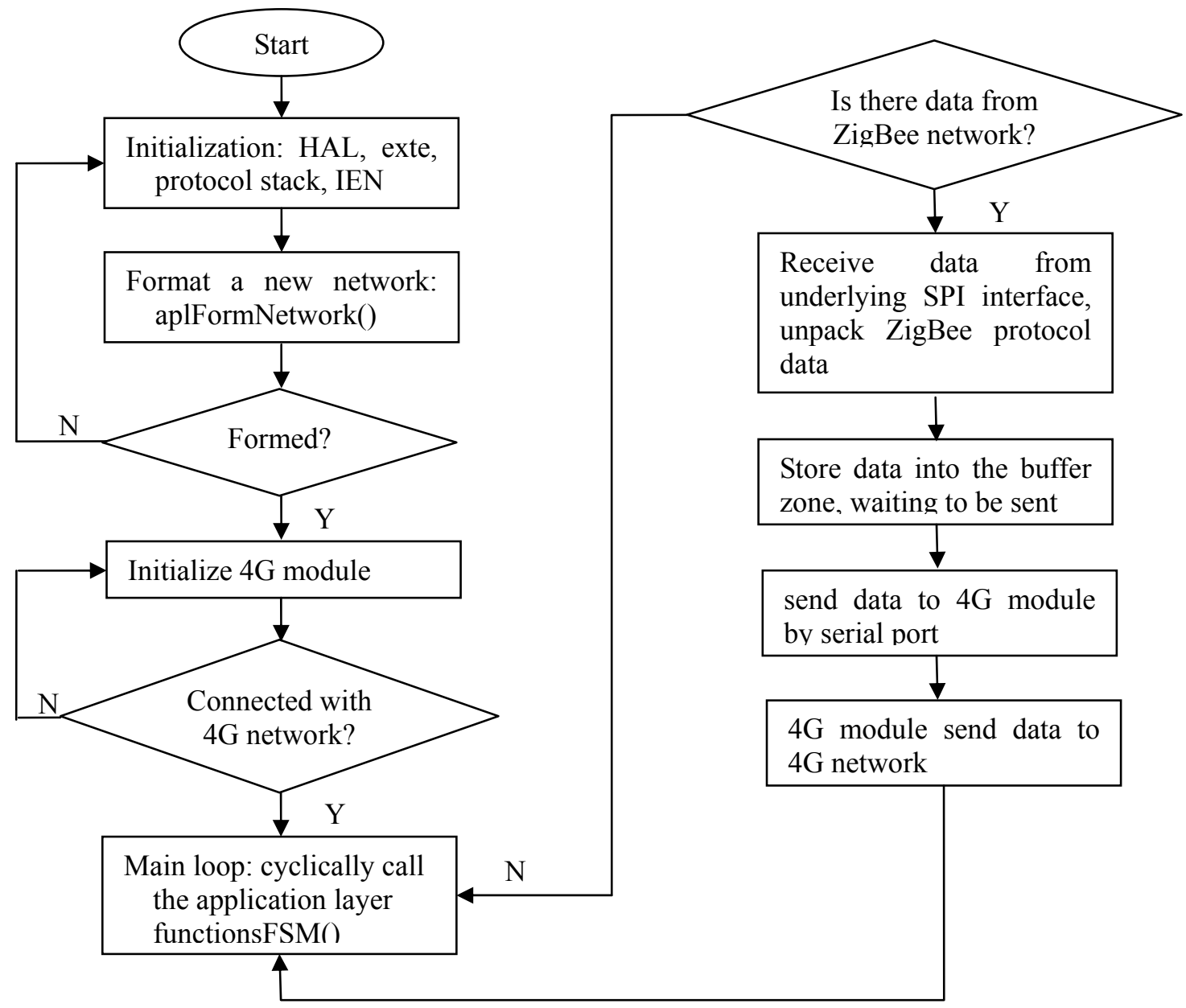

Fig.4. Flow chart of data exchanges between coordinator and 4G module 


\section{B. Design of $4 G$ communication software}

The main task of coordinator node when network is built is responsible for transmitting data of monitoring network to $4 \mathrm{G}$ network and being conversion gateway based on ZigBee protocol and 4G protocol. It has two sorts of work mode, that is, downlink and data data uplink. In the former mode, the $4 \mathrm{G}$ module transmits real-time the commands of monitoring server to monitoring network to complete the specific data collection and control tasks in accordance with these commands. In the later mode, the $4 \mathrm{G}$ module transmits multi-sensor data periodical collected by monitoring network to monitoring server, according to time interval.

C. Design of monitoring server system software

The monitoring system will trigger an alarm automatically when potential fire hazards or forest fire are found, and help the administrative department of the forest to adopt the effective measures to stop fire hazards stretching in the forest.

This paper designs the monitoring server system as shown in Fig.5.

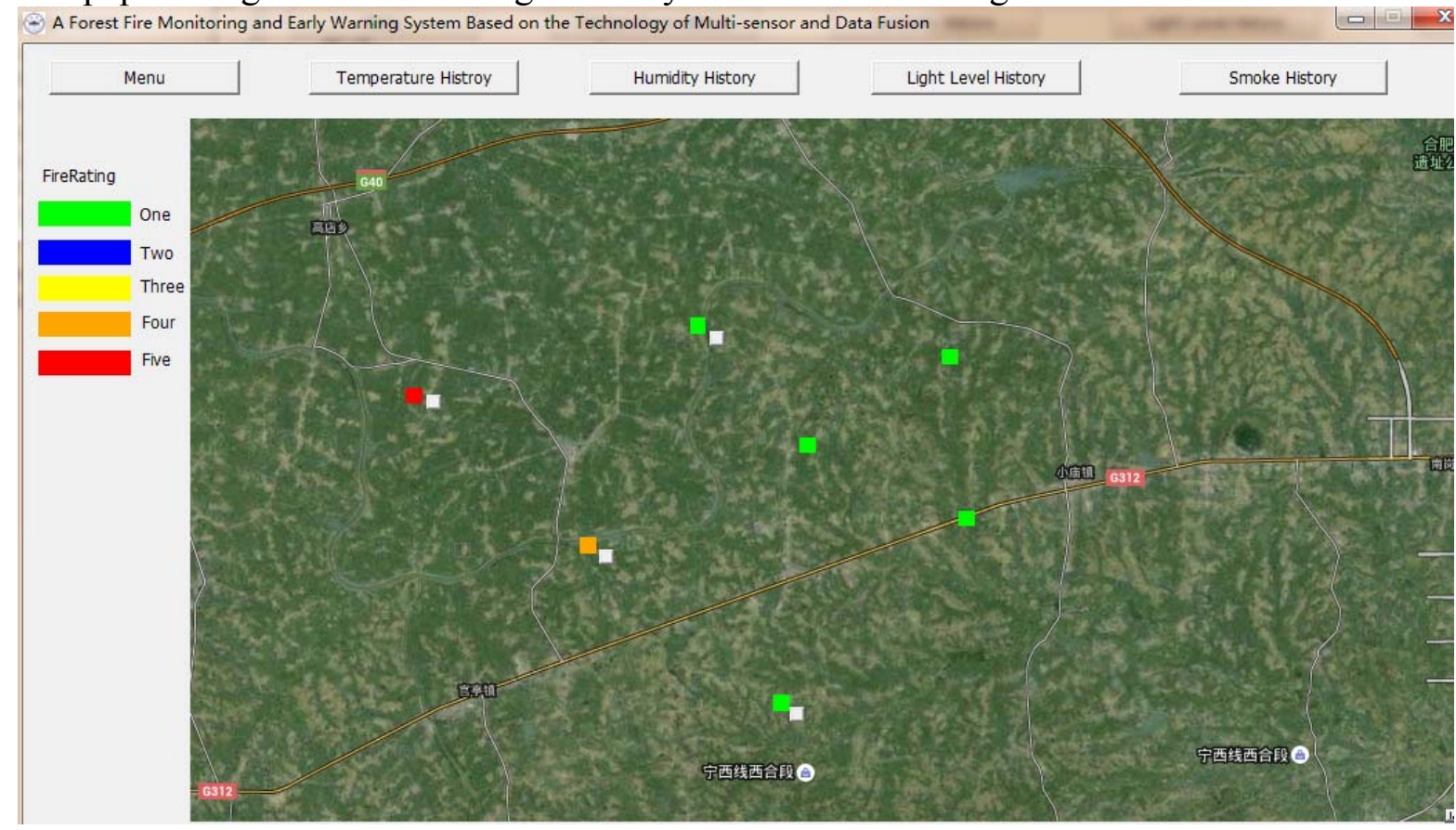

Fig.5. Monitoring server system main interface

We can see the whole forest fire situation in the main interface, and fire danger rating distribution. When we want to understand the specific circumstances of a particular node information, we can enter into the child interface. On the child interface, it's convenient to monitor the node's real-time information such as current temperature, relative humidity, light level, smoke concentration and flame situation. And the main interface can also display historical trend curve of forest fire factors mentioned above, set alarm value, alarm when fire factors data are abnormal, display data transmission rates and state of connection between monitoring nodes and the server.

\section{Conclusion}

Wireless sensor network has a great impact on our future life in many fields for its feature of low power lost, low cost, dynamic multi-routing, protocol flexibility, self-organization and real-time monitoring. This paper designs a forest fire monitoring and early warning system based on the technology of multi-sensor and multilevel data fusion. Furthermore, the specific design methods of multilevel hierarchical clustering protocol and $4 \mathrm{G}$ network in the forest fire monitoring system is proposed. The monitoring system is good at flexible structure, low one-time cost, easy operation, wide expansion and better promotional value when compared to traditional forest fire monitoring system. It will also be beneficial to the study and exploration of the forest environmental monitoring and promote more applications and development of wireless sensor network in future. 


\section{Acknowledgement}

In this paper, the research was sponsored by by the National Natural Science Foundation of China (61172127) and the 211 Project of Anhui University (KJQN1121).

\section{References}

[1] Guozhu Wang, Junguo Zhang, Wenbin Li, Dongxu Cui, Ye Jing. A Forest Fire Monitoring System Based on GPRS and ZigBee Wireless Sensor Network[C].2010 5th IEEE Conference on Industrial Electronics and Applicationsis.

[2] Fang Wang. Application of IPSTAR satellite communications system for rapid Disposal of forest and grassland fire. Satellite and Network, 2008,10:45, 10:45-49

[3] Fei Qin, Tao Feng. Wireless sensor networks in field application[J], Application of Electronic Technique, 2007 (9): 10-12

[4] Cornelissen, S.A.. MEMS deformable mirror actuators with enhanced reliability [J].Proceedings of SPIE - The International Society for Optical Engineering, 2012.

[5] Yuenew Jiang. Application prospect of compass satellite navigation and communication system for forest fire[J]. Satellite and Network, 2008,12:42-45

[6] Sekkas, Odysseas \& Hadjieftymiades, Stathes \& Zervas, Evangelos. A multi-level data fusion approach for early fire detection [C]. Proceedings - 2nd International Conference on Intelligent Networking and Collaborative Systems,2010: 479-483

[7] Bihua Zhu, Daqing Zhu. Automatic Monitoring System for Forest Fire Based on Wireless Sensor Network[C]. Advanced Materials Research Vols 694-697 (2013) pp 1211-1214

[8] Liyang Yu, Neng Wang, Xiaoqiao Meng. Real-time Forest Fire Detection with Wireless Sensor Networks[C].Wireless Communications, Networking and Mobile Computing, 2005.

[9]Ahmed Helmy helmy, Brendan Jennings, Liam Murphy, Tom Pfeifer. Towards autonomic handover decision management in 4G networks[C]. 9th IFIP/IEEE International Conference on Management of Multimedia and Mobile Networks and Services, MMNS 2006, Dublin, Ireland, October 25-27, 2006. Proceedings

[10]Wairagu G. Richard. Extending LEACH routing algorithm for wireless sensor networks[J]. A Project Report Submitted to the School of Graduate Studies in Partial Fulfillment for the Award of Master of Science in Data Communication and Software Engineering Degree of Makerere University, March, 2009. 